\title{
Estudio sobre la resistencia quimica de baldosas ceramicas no-esmaltadas para pavimentos industriales
}

\author{
L.ESCODA, M.LLEDÓ, J.J.SUÑOL, P.ROURA, J.CARDA* \\ Departament de Física, Universitat de Girona, Girona. \\ Escola Superior de Tecnologia i Ciències Experimentals, Universitat Jaume I, Castelló de la Plana.
}

\begin{abstract}
La resistencia al ataque químico de baldosas cerámicas está contemplada en la Norma UNE-EN ISO 10545-13. Dicha norma establece una serie de ensayos a temperatura ambiente en los cuales se analiza visualmente el efecto sobre el aspecto de la superficie de disoluciones acuosas de diversos productos químicos. Según esta norma, las baldosas objeto de nuestro estudio están clasificadas como de máxima resistencia (UHA). Sin embargo, las condiciones a las que pueden verse sometidas pueden ser considerablemente más agresivas que las contempladas en la Norma, por lo que es necesario un estudio sistemático. En el presente trabajo, se ha diseñado un procedimiento para evaluar el efecto de diversas disoluciones acuosas de diversos ácidos orgánicos e inorgánicos. Las probetas sumergidas en las diferentes soluciones han estado sometidas a las siguientes condiciones: $\mathrm{T}=60^{\circ} \mathrm{C} ; \mathrm{pH}=2$ y procesos de agitación. El posible ataque químico de la superficie de las baldosas se ha evaluado por observación visual así como por microscopía óptica y microscopía electrónica de barrido (SEM). Complementariamente se ha analizado por absorción atómica la eventual disolución de diversos iones metálicos en el sistema $\mathrm{M}$ (III)-L(M = Fe; $\mathrm{L}$ $=\mathrm{H}_{2} \mathrm{O}$ o L $=$ ligando). El análisis ha revelado en todas las soluciones la disolución progresiva de pequeños precipitados de óxidos de hierro presentes en el cuerpo cerámico.
\end{abstract}

Palabras clave: baldosas cerámicas, corrosión, microscopía electrónica

Study on the chemical resistance of ceramic tiles no enamelled for industrial pavement

The chemical resistance of ceramic tiles is the subject of the European Standard UNE-EN ISO 10545-13. In order to evaluate the effect of aqueous solutions of several chemicals agents on the aspect of the tile surface, this standard establishes a series of tests at room temperature followed by visual inspection. According to this standard the tiles of this study are classified as being of maximum resistance (UHA). However operating conditions can be more aggressive than those detailed in the standard. So, a systematic study has been undertaken.

In the present work, the effect of aqueous solutions of several organic and inorganic acids on the tile surface is evaluated. Samples immersed in different solutions are subjected to the following conditions: $\mathrm{T}=60^{\circ} \mathrm{C} ; \mathrm{pH}=2$ and to agitation processes. Visual analysis, as well as optical microscopy and scanning electron microscopy (SEM) were performed in order to determine the possible variation of the superficial aspect of tiles. Moreover, atomic absorption spectrophotometry has been used in order to obtain quantitative information concerning the solubility of system $\mathrm{M}$ (III)- $\mathrm{L}\left(\mathrm{M}=\mathrm{Fe}\right.$; $\mathrm{L}=\mathrm{H}_{2} \mathrm{O}$ or $\mathrm{L}=$ ligand). The results obtained show, in all cases, a progressive dissolution of iron oxide precipitates presents in the ceramic body.

Keywords: ceramic tiles, corrosion, SEM

\section{INTRODUCCIÓN}

Los materiales cerámicos constituyen, en conjunto, la familia de materiales que presentan mayor resistencia química en un amplio espectro de condiciones agresivas. Sin embargo, no se trata de materiales inertes. La problemática de su corrosión en medio ácido a temperatura superior a la ambiente ha sido objeto de recientes estudios [1-2]. La estabilidad de los compuestos presentes en función del $\mathrm{pH}$ ha sido estudiada por diferentes autores [3-4].

Existe una clasificación normalizada de la resistencia química de baldosas cerámicas (UNE-EN ISO 10545-13). Según esta norma, la clasificación se realiza tomando como base el cambio de aspecto de las baldosas después de ser parcial- mente sumergidas durante 24 horas a temperatura ambiente en diversas disoluciones agresivas en reposo. El parámetro principal que se toma como criterio de agresividad de las disoluciones es el $\mathrm{pH}$. En el caso de medios ácidos se utiliza exclusivamente $\mathrm{HCl}$ con lo que no se toma en consideración el posible carácter oxidante o bien ligando de determinados ácidos. Por otra parte, el estado de movimiento de la disolución o bien la temperatura también pueden acentuar el carácter agresivo [5]. Desde el punto de vista del análisis de los efectos sobre la cerámica, la Norma se basa en una evaluación visual del cambio de aspecto. Este criterio puede resultar especialmente limitado en el caso de cerámicas no esmaltadas en las cuales la 
rugosidad propia de la superficie puede enmascarar procesos de solubilización que podrían resultar importantes en exposiciones prolongadas al medio agresivo. El uso de técnicas como la microscopía electrónica de barrido (SEM) con microanálisis (EDX), o la difracción de rayos $X$, permiten un análisis más exhaustivo [6-7]

El objetivo del presente trabajo es analizar la resistencia química de baldosas cerámicas no esmaltadas clasificadas según la Norma como de resistencia química máxima. Se utilizan principalmente para pavimentos industriales. Por ello se ha analizado el efecto de un amplio abanico de disoluciones que cubre una fracción importante de las posibles condiciones de trabajo. De hecho, dado su carácter más sistemático, nos limitaremos a la descripción de los resultados obtenidos en medio ácido. El eventual cambio de aspecto se ha observado por técnicas microscópicas y además se ha evaluado la solubilización de algunos iones metálicos con técnicas analíticas. Además, se está analizando el comportamiento dilatométrico de estas baldosas [8].

\section{MÉTODO EXPERIMENTAL}

Las baldosas cerámicas presentan la composición química y mineralógica detalladas en la Tabla I. Se han empleado las técnicas de fluorescencia de rayos $\mathrm{X}$ y difracción de rayos $\mathrm{X}$. Se trata de un material con un elevado contenido en mullita $(65$ $\%)$, una fracción importante de la cual ya se ha introducido en la pasta. La presencia de una elevada densidad de partículas inertes de mullita durante la cocción da como resultado una elevada porosidad cerrada (aproximadamente un $35 \%$ en la mullita). La figura 1 muestra el difractograma correspondiente a la baldosa cerámica, en éste se constata la presencia de cuarzo y mullita como fases mayoritarias. Las diferentes fases se distinguen claramente por microscopía electrónica de barrido (SEM) con la ayuda de una sonda de microanálisis. En la figura 2, se presentan micrografías típicas que revelan tanto la elevada porosidad como la presencia de precipitados
TABLA I: ANÁLISIS COMPOSICIONAL Y MINERALÓGICO DE LA CERÁMICA OBJETO DEL ESTUDIO.

\begin{tabular}{|c|c|c|}
\hline \multicolumn{3}{|c|}{ COMPOSICION QUIMICA } \\
\hline $57.1 \mathrm{SiO}_{2}-34.7 \mathrm{Al}_{2} \mathrm{O}_{3}-$ & $1.72 \mathrm{~K}_{2} \mathrm{O}-1.29 \mathrm{Fe}$ & $\mathrm{O}_{3}-3.41 \mathrm{Na}_{2} \mathrm{O}-0.23 \mathrm{CaO}$ \\
\hline \multicolumn{3}{|c|}{ ANALISIS MINERALOGICO } \\
\hline & $\begin{array}{l}\text { CUARZO } \\
\text { Cristobalita (opal) } \\
\text { Mullita } \\
\text { Fase vitrea } \\
\quad \mathrm{Fe}_{2} \mathrm{O}_{3} 1.3 \%\end{array}$ & $\begin{array}{l}15 \% \mathrm{~F}_{\mathrm{Q}} \\
10 \% \\
65 \% \\
10 \%\end{array}$ \\
\hline \multicolumn{3}{|l|}{ POROSIDAD } \\
\hline Porosidad en la mullita: & & $\sim 35 \%$ \\
\hline
\end{tabular}

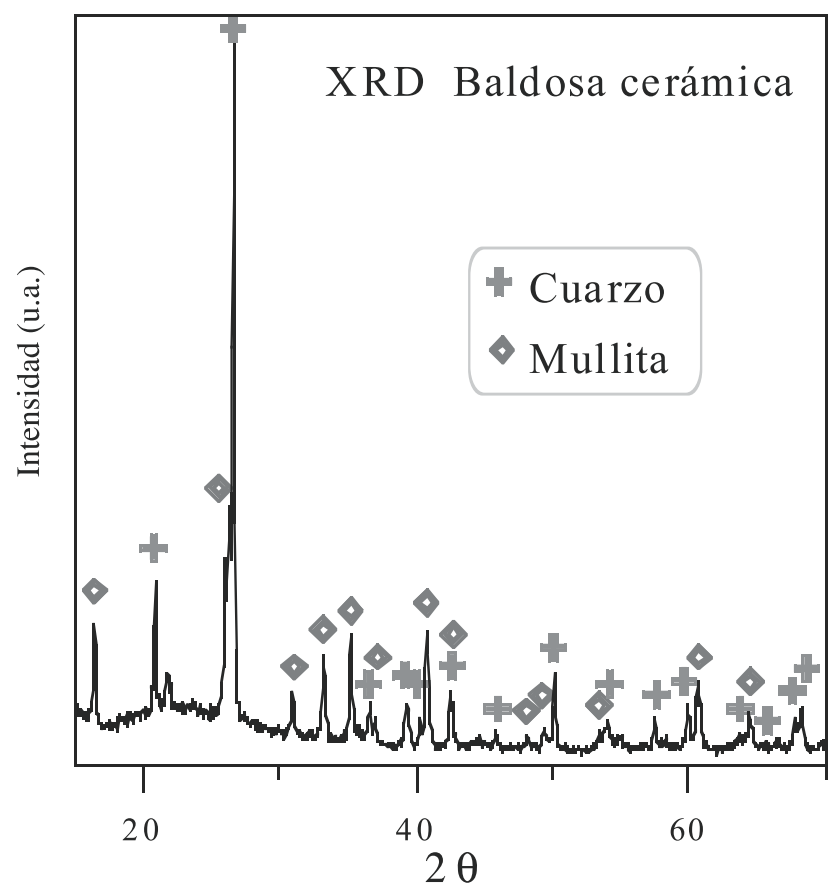

Figura 1: Difractograma de la cerámica.
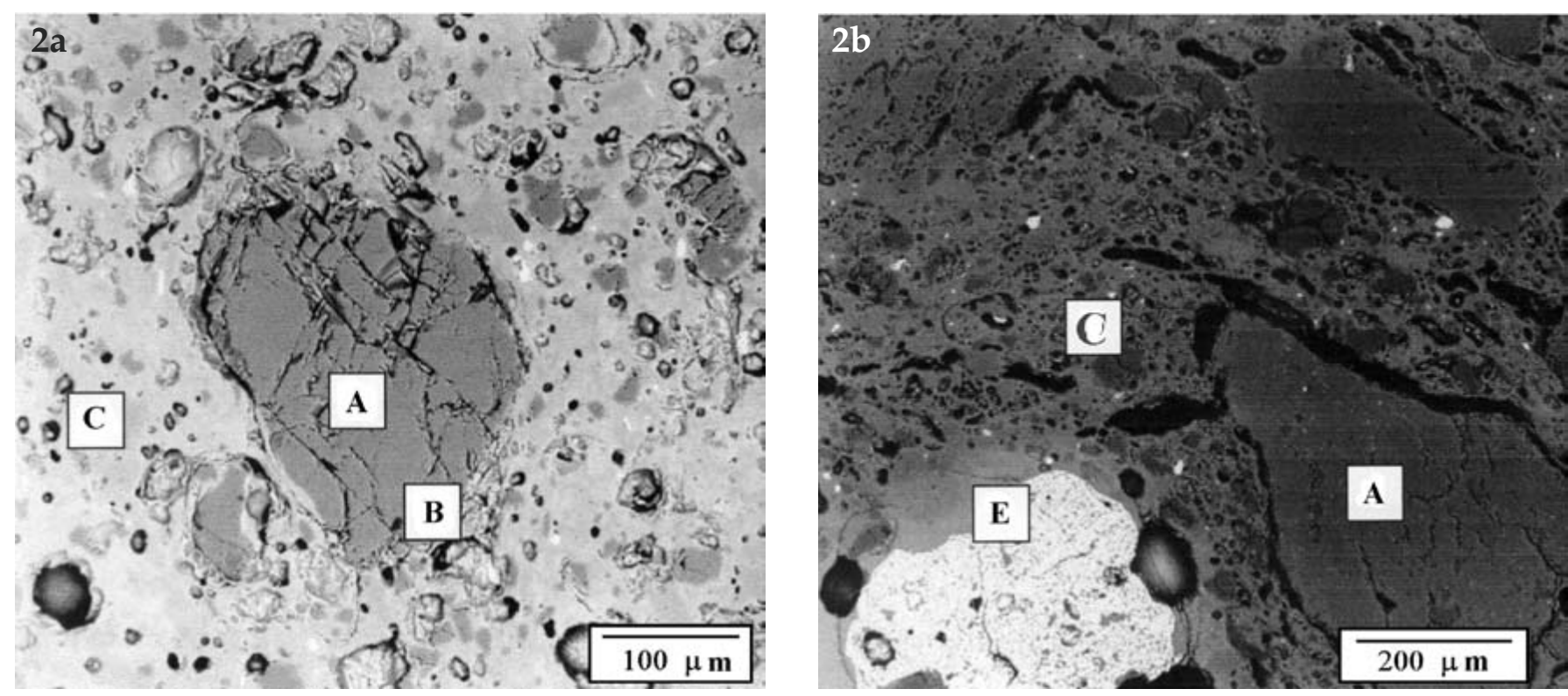

Figura 2: Micrografías obtenidas por SEM con electrones retrodifundidos. En la figura (2 a) se distingue claramente un grano de cuarzo (A) separado de la matriz de mullita (C) por una fase vítrea (B). En la figura (2 b), se distingue, además, un precipitado de óxido de hierro (E). En ambas micrografías se aprecia una elevada poricidad cerrada. 
de óxido de hierro. Su fracción volumétrica se ha determinado por análisis de un gran número de micrografías como la de la figura 2. Aparentemente, tales precipitados aparecen durante la cocción.

Se han cortado probetas de dimensiones $5 \times 5 \times 1,5 \mathrm{~cm}$, las cuales se han sumergido parcialmente durante $24 \mathrm{~h}$ en las disoluciones de ataque. La parte no sumergida se ha protegido de los vapores de la disolución recubriéndola con una capa de resina. Ello permite, una vez extraída la probeta de la disolución, determinar con mayor precisión los eventuales cambios de aspecto.

Los ácidos empleados cubren un amplio abanico. Los ácidos ensayados se clasifican en función de sus características oxidantes y como agentes ligandos de cationes metálicos. Como ácido oxidante se analiza el comportamiento de los ácidos nítrico $\left(\mathrm{HNO}_{3}\right)$ y sulfúrico $\left(\mathrm{H}_{2} \mathrm{SO}_{4}\right)$. Los ácidos escogidos por sus características complejantes son, fundamentalmente, orgánicos. El ácido clorhídrico, $\mathrm{HCl}$, es normativo. Todas las soluciones se ajustan a $\mathrm{pH}=2$ mediante ácido clorhídrico concentrado. La temperatura de inmersión se mantiene en $60^{\circ} \mathrm{C}$ empleando un baño termostático y en continua agitación mecánica para garantizar su uniformidad. La agitación de las soluciones de los ácidos facilita, también, el proceso de extracción y disolución de los óxidos metálicos. Una vez transcurridas $24 \mathrm{~h}$ las probetas se sacaban de las disoluciones y se limpiaban siguiendo los procedimientos detallados en la Norma. Tras eliminar la resina, se procedía a su observación visual y a la comparación con el blanco. Dada la existencia de los precipitados de óxido de hierro los cuales eran a priori más susceptibles al ataque químico, se procedió a marcar su posición antes del ataque con lápiz de grafito. Ello permitió comparar su aspecto por microscopía óptica antes y después del ataque. En los casos en que se detectó algún cambio se pasó a la observación por SEM.

La concentración de iones disueltos se cuantificó por absorción atómica y por ICP (Plasma de Acoplamiento Inductivo) tras comparar las disoluciones problema con disoluciones patrón. Para determinar exclusivamente Fe es suficiente una sola técnica de análisis. No obstante, el empleo de ambas permite contrastar la fiabilidad y exactitud de los resultados. Cada ensayo se realizó por duplicado y se promedió la concentración obtenida en cada caso.

\section{RESULTADOS}

En ningún caso la observación visual reveló el más tenue cambio de aspecto aún en las condiciones más agresiva utilizadas. Tampoco la microscopía óptica o SEM fueron capaces de revelar ningún cambio morfológico en las fases de silicatos (mullita, cuarzo o fases vítreas). En cambio, sí que algunos de los gránulos de óxido de hierro aparecían con diversos grados de disolución. Como muestra podemos ver en la figura 3, cómo los gránulos que se encuentras fuera de la zona previamente protegida por la resina, están parcialmente disueltos. Sin embargo, no es posible por microscopía cuantificar el grado de disolución.

Las Tablas II y III resumen la concentración del ión $\mathrm{Fe}^{3+}$ que ha pasado a las diferentes disoluciones. Tal y como ya apuntaban las observaciones por microscopía, el $\mathrm{Fe}^{3+}$ aparece en concentraciones significativas, procedente de los precipitados de óxido.

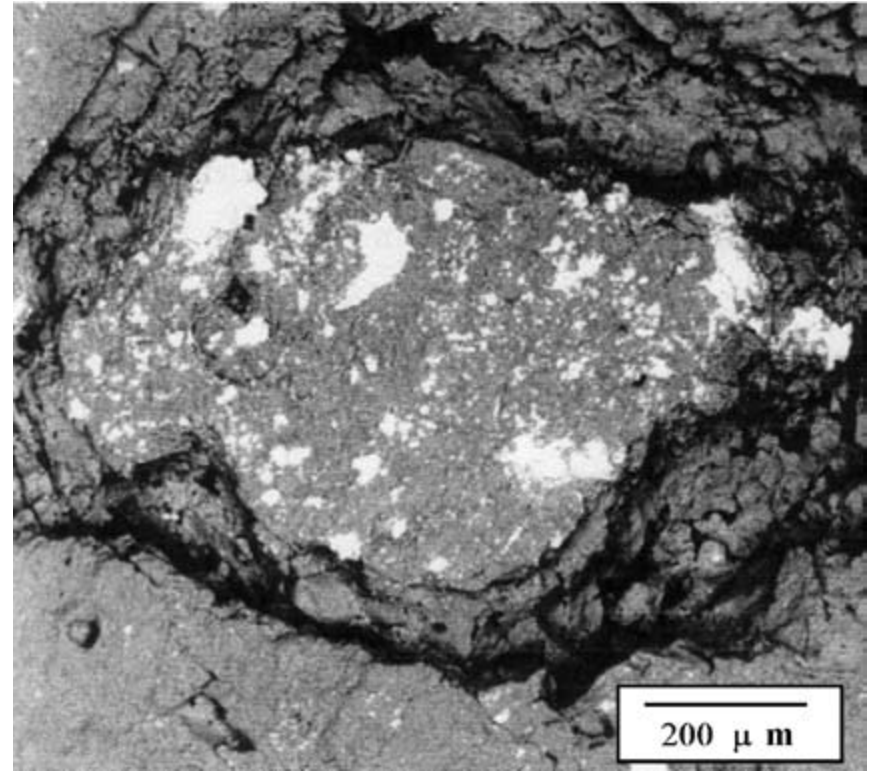

Figura 3: Micrografía SEM típica del aspecto de un precipitado de óxido de hierro tras su disolución, casi completa, en medio ácido. El óxido de hierro que aún permanece aparece brillante.

TABLA II: CONCENTRACIÓN DE IONES DISUELTOS EN LOS DIVEROS MEDIOS DE ATAQUE. EN EL CASO DEL FE ${ }^{3+}$ SE HA CALCULADO EL GROSOR EQUIVALENTE DE PRECIPITADO DISUELTO.

\section{ACIDOS ORGANICOS (ACOMPLEJANTES)}

\begin{tabular}{|c|c|c|}
\hline Reactivo químico & {$\left[\mathrm{Fe}^{3+}\right](\mathrm{ppm})$} & Grosor capa $(\mu \mathrm{m})$ \\
\hline Ácido acético & 0,257 & 0,116 \\
\hline Ácido fórmico & 0,515 & 0,234 \\
\hline Ácido tartárico & 0,400 & 0,181 \\
\hline Ácido láctico & 0,238 & 0,108 \\
\hline Ácido oxálico & 0,125 & 0,057 \\
\hline
\end{tabular}

2. ACIDOS INORGANICOS

\begin{tabular}{|c|c|c|}
\hline Reactivo químico & {$\left[\mathrm{Fe}^{3+}\right](\mathrm{ppm})$} & Grosor capa $(\mu \mathrm{m})$ \\
\hline Ácido clorhídrico & 0,684 & 0,261 \\
\hline Ácido nítrico & 0,434 & 0,198 \\
\hline Ácido sulfúrico & 0,579 & 0,183 \\
\hline
\end{tabular}

Una imagen más clara de lo que significan las concentraciones de $\mathrm{Fe}^{3+}$ la podemos obtener como sigue. A partir de la concentración y volumen de la disolución calculamos el número de iones disueltos, N. Si consideramos que provienen exclusivamente de los precipitados podemos calcular el grosor que se ha disuelto en promedio aplicando la fórmula siguiente:

$$
\text { grosor }=\frac{N P_{m}}{2 S f_{s} \rho}
$$

donde, $\rho$ es la densidad del óxido férrico $\left(5,7 \mathrm{~g} / \mathrm{cm}^{3}\right)$ y $\mathrm{P}_{\mathrm{m}}$ su peso molecular $(160 \mathrm{~g} / \mathrm{mol}), \mathrm{S}$ la superficie expuesta a la disolución $\left(22 \mathrm{~cm}^{2}\right)$ y $\mathrm{f}_{\mathrm{s}}$ la fracción de esta superficie correspondiente a los precipitados (1,3\%). En todos los casos vemos que el grosor de la capa disuelta es del orden o inferior a 0,2 $\mu \mathrm{m} /$ día. 


\section{ANÁLISIS}

Tanto los resultados de microscopía como la cuantificación de los iones disueltos revelan que la fase más vulnerable de la cerámica son los óxidos de hierro. La disolución del $\mathrm{Fe}_{2} \mathrm{O}_{3}$ se puede considerar, únicamente, por las condiciones ácidas de trabajo $(\mathrm{pH} \approx 2)$ de todos los reactivos ensayados. Una vez extraído el óxido y disociado, el catión metálico $\mathrm{Fe}^{3+}$ es soluble en las condiciones termodinámicas de las soluciones problema como muestra el diagrama de especiación (figura 4) del catión $\mathrm{Fe}^{3+}$ en función del $\mathrm{pH}$ en medio acuoso. Este diagrama implica que los óxidos de hierro (III) hidratados son insolubles en medio acuoso a $\mathrm{pH}>2.5$ [9] Si comparamos los ácidos inorgánicos vemos que no existe diferencia significativa de comportamiento entre el $\mathrm{HNO}_{3}$ y el $\mathrm{H}_{2} \mathrm{SO}_{4}$ es decir, el carácter oxidante del $\mathrm{HNO}_{3}$ no tiene efecto sobre la disolución del $\mathrm{Fe}^{3+}$ lo cual significa que la totalidad del Fe se encuentra en su estado máximo de oxidación en el cuerpo cerámico. La mayor concentración de $\mathrm{Fe}^{3+}$ detectada en la disolución que contiene, únicamente, $\mathrm{HCl}$ comparada con los otros ácidos inorgánicos, se fundamenta en el proceso en que este ácido es el único que forma un complejo con el $\mathrm{Fe}^{3+}\left(\mathrm{FeCl}^{4-}\right)$. No obstante, sus resultados son comparables con los obtenidos por la mayoría de ácidos inorgánicos.

Las condiciones de acidez del medio, originan un grado de solubilización importante en los ácidos orgánicos, a pesar de considerarse químicamente como ácidos más débiles. La secuencia de reacciones que tienen lugar en medio acuoso en condiciones ácidas se pueden resumir: el $\mathrm{Fe}^{3+}$ extraído se mantiene en disolución debido a un proceso de solvatación que origina la formación de óxidos de hierro (III) hidratados solubles en las condiciones de trabajo (1) y, la posterior hidrólisis de éstos (2) atendiendo a su comportamiento como ácido de Pearson:

$\mathrm{Fe}_{2} \mathrm{O}_{3(\mathrm{~s})}+\mathrm{n} \mathrm{H}_{2} \mathrm{O} \Rightarrow \mathrm{Fe}_{2} \mathrm{O}_{3} \cdot \mathrm{n} \mathrm{H}_{2} \mathrm{O}_{(\mathrm{aq})} ; \mathrm{pH}<2$

$\left.\mathrm{Fe}_{2} \mathrm{O}_{3} \cdot \mathrm{nH}_{2} \mathrm{O}_{(\text {aq) }}+\mathrm{H}_{2} \mathrm{O} \Leftrightarrow \mathrm{Fe}\left(\mathrm{H}_{2} \mathrm{O}\right)_{\mathrm{n}-1}(\mathrm{OH})\right]^{2+}{ }_{(\mathrm{aq})}+\mathrm{H}_{3} \mathrm{O}^{+} ; \mathrm{pH} \approx 2$

En los ácidos orgánicos, que contienen grupos funcionales carboxílicos, los equilibrios de formación de especies complejas con los diferentes ligandos orgánicos compiten con los equilibrios de hidrólisis. La estabilidad de estos complejos, en medio acuoso, en función del $\mathrm{pH}$ ha sido estudiada por diferentes autores [10-13]. En el presente trabajo todos los ácidos carboxilicos ensayados tenían afinidad por el Fe(III) a diferentes $\mathrm{pH}$, con lo cual se incrementa su solubilidad.

\section{CONCLUSIONES}

Se ha analizado la resistencia química de una baldosa cerámica no esmaltada ampliando las disoluciones contempladas en la Norma ISO-EN-10545-13 y sometiéndolas a condiciones más agresivas de $\mathrm{pH}$, temperatura y estado de agitación. En

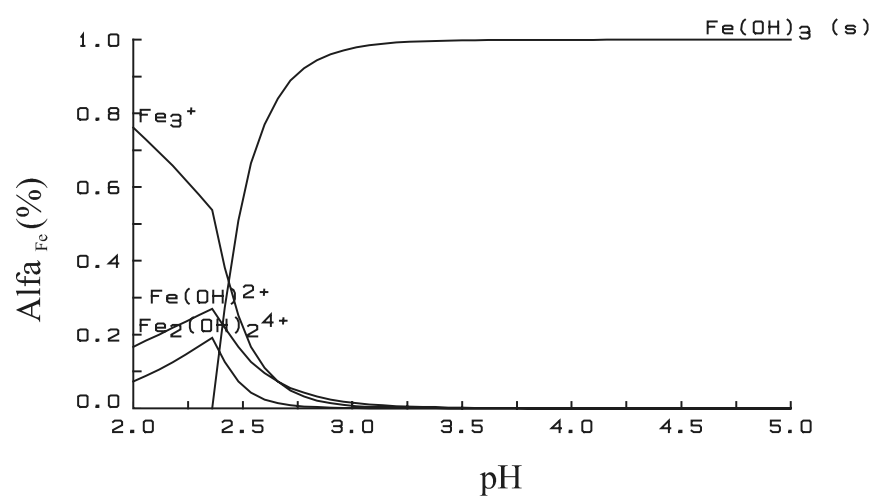

Figura 4: Representación del diagrama de especiación del $\mathrm{Fe}^{3+}$, en medio acuoso, en función del $\mathrm{pH}$. La figura indica que a $\mathrm{pH}<2.2$ todo el $\mathrm{Fe}^{3+}$ se encuentra en solución formando diferentes especies químicas que se encuentran en equilibrio. El modelo representado en la figura esta formado por tres especies $\left(\mathrm{Fe}^{3+}, \mathrm{Fe}(\mathrm{OH})^{2+}\right.$ y $\left.\mathrm{Fe}_{2}(\mathrm{OH})_{2}{ }_{2}^{4+}\right)$. A $\mathrm{pH}$ comprendido entre 2.2 y 2.4 empieza a formarse el precipitado de $\mathrm{Fe}(\mathrm{OH})_{3}$. Esta especie es la única existente a $\mathrm{pH}>3.20$.

ningún caso la observación visual establecida en la Norma ha revelado ningún cambio de aspecto. Sin embargo, al análisis por microscopía así como la cuantificación de los iones metálicos $\left(\mathrm{Fe}^{3+}\right)$ disueltos indican claramente que la cerámica no es inerte a ninguna de la disoluciones utilizadas. El elemento más vulnerable de la microestructura lo constituyen los precipitados de óxido de hierro. Por otra parte, las fases de silicatos no han sufrido disolución en grado mesurable.

\section{BIBLIOGRAFÍA}

1. M. Schacht, N. Boukis and E. Dinjus, Journal of Materials Science, 2000, 35, 6251.

2. N.S. Jacobson, E.J. Opila and K.N. Lee, Current opinion in Solid State \& Materials Science, 2001, 5, 301.

3. V. Salvadó, X. Ribas, V. Zelano, G. Ostacoli and M. Valiente, Polyhedrom, $1989,8,813$

4. J.J. Suñol, L. Escoda, J. Saurina and J. Arnau, IX Meditarrean Congress of Chemical Engineering, Barcelona, 2002, 175.

5. L. Escoda, Thesis, Universitat de Girona, 2001.

6. S.S. Ramos, F.B. Reig, J.V.G. Adelantado, D.J.Y. Marco and A.D. Carbó, Spectroquimica Acta B-Atomic Spectroscopy, 2002, 57, 689.

7. S.S. Ramos, F.B. Reig, J.V.G. Adelantado, D.J.Y. Marco and A.D. Carbó, Analytical and bioanalytical Chemistry, 2002, 373, 893.

8. L. Escoda, J.J. Suñol, M. Lledó, P. Roura, J. Cardà, VII European Conference on Ceramics, Génova, 2000, 74

9. V. Salvadó, X. Ribas, M. Blanco and M. Valiente, Inorg. Chim. Acta 1987, 137,155

10. L. Escoda, V. Salvadó and F. De la Torre, Polyhedrom, 1999, 18, 3275

11. L. Escoda, V. Salvadó and F. De la Torre, Polyhedrom, 1999, 18, 3269

12. V. Salvadó, X. Ribas and M. Valiente, J. Chem. Res. (M) 1989, 2533

13. V. Salvadó, X. Ribas and M. Valiente, Polyhedrom. 1990, 22, 2675

Recibido: 11.01 .01

Aceptado: 28.02 .03 